\title{
New approach to assess sperm DNA fragmentation dynamics: Fine-tuning mathematical models
}

\author{
Isabel Ortiz ${ }^{1 *}$ (D), Jesús Dorado ${ }^{1}$, Jane Morrell ${ }^{2}$, Jaime Gosálvez ${ }^{3}$ Francisco Crespo ${ }^{4}$, Juan M. Jiménez ${ }^{5}$ and \\ Manuel Hidalgo ${ }^{1}$
}

\begin{abstract}
Background: Sperm DNA fragmentation (sDF) has been proved to be an important parameter in order to predict in vitro the potential fertility of a semen sample. Colloid centrifugation could be a suitable technique to select those donkey sperm more resistant to DNA fragmentation after thawing. Previous studies have shown that to elucidate the latent damage of the DNA molecule, sDF should be assessed dynamically, where the rate of fragmentation between treatments indicates how resistant the DNA is to iatrogenic damage. The rate of fragmentation is calculated using the slope of a linear regression equation. However, it has not been studied if sDF dynamics fit this model. The objectives of this study were to evaluate the effect of different after-thawing centrifugation protocols on sperm DNA fragmentation and elucidate the most accurate mathematical model (linear regression, exponential or polynomial) for DNA fragmentation over time in frozen-thawed donkey semen.

Results: After submitting post-thaw semen samples to no centrifugation (UDC), sperm washing (SW) or single layer centrifugation (SLC) protocols, SDF values after $6 \mathrm{~h}$ of incubation were significantly lower in SLC samples than in SW or UDC. Coefficient of determination $\left(R^{2}\right)$ values were significantly higher for a second order polynomial model than for linear or exponential. The highest values for acceleration of fragmentation (aSDF) were obtained for SW, followed by SLC and UDC.

Conclusion: SLC after thawing seems to preserve longer DNA longevity in comparison to UDC and SW. Moreover, the fine-tuning of models has shown that sDF dynamics in frozen-thawed donkey semen fit a second order polynomial model, which implies that fragmentation rate is not constant and fragmentation acceleration must be taken into account to elucidate hidden damage in the DNA molecule.
\end{abstract}

Keywords: Colloid centrifugation, Dynamics, Fine-tuning, Mathematical models, Sperm DNA fragmentation

\section{Background}

The importance of the assessment of sperm chromatin to predict the potential fertility has been shown in humans and animals [1-5]. The crucial role that sperm DNA fragmentation (sDF) plays in sperm analysis is due to its relationship with infertility problems in spite of apparently normal values for routine sperm parameters such as motility, morphology or integrity of sperm membranes [6]. The assessment of this parameter is even more critical when sperm quality is limited or compromised, as happens

\footnotetext{
* Correspondence: v52orjai@uco.es

${ }^{1}$ Veterinary Reproduction Group, Department of Animal Medicine and Surgery, Faculty of Veterinary Medicine, University of Cordoba, 14071 Cordoba, Spain

Full list of author information is available at the end of the article
}

in some subfertile males, or in cool-shipped or frozenthawed semen samples [7]. Therefore, it becomes of the utmost importance to select spermatozoa with intact DNA in order to achieve a higher success in pregnancy rates [8].

Previous studies have evaluated the effect of different centrifugation techniques to select frozen-thawed donkey sperm $[9,10]$ concluding that, although sperm quality was improved when colloid centrifugation was performed, this procedure did not select intact DNA spermatozoa when performing a static analysis of sDF (baseline value). Nevertheless, it has been shown in several studies $[11,12]$ that a dynamic assessment of $\mathrm{sDF}$ is more accurate to simulate ex vivo sperm maintenance and to evaluate latent chromatin damage than only 
considering baseline values. In these studies semen samples were submitted to thermal stress and SDF values were recorded at different times. Subsequently, a linear regression equation was calculated and the rates of fragmentation (the slope of the linear regression equation, sDF\%/time) of the treatments were compared.

This approach solved the issue of the cryptic DNA damage; however, another question arose: do DNA dynamics fit to a linear regression model? Although linear regression is the simplest model, it entails that DNA damage is a simple process with constant speed. Before accepting this statement as an actual fact, a fine-tuning of mathematical models for DNA fragmentation over time should be carried out. Thus, the objectives of this study were to evaluate the effect of different afterthawing centrifugation protocols on sperm DNA fragmentation and elucidate the most accurate mathematical model for describing DNA fragmentation dynamics in frozen-thawed donkey semen.

\section{Methods}

\section{Animals and semen collection}

Six healthy Andalusian donkeys (aged from 6 to 15) were used for this study. Semen collection was performed using a Missouri artificial vagina with an in-line gel filter (Minitüb, Tiefenbach, Germany) in the presence of a jenny in natural or induced estrus. Three ejaculates per animal were collected, obtaining a total number of 18 ejaculates. All animal procedures were performed in accordance with the Spanish laws for animal welfare and experimentation.

\section{Sperm freezing and thawing}

Sperm was frozen and thawed following the methodology described by Ortiz et al. [10]. Briefly, seminal plasma was removed by centrifugation $(400 \times g$ for $7 \mathrm{~min})$ and the sperm pellet was resuspended with a commercial freezing medium containing egg-yolk and glycerol (Gent; Minitüb, Tiefenbach, Germany). Then, semen was slowly cooled for $2 \mathrm{~h}$, loaded into $0.5 \mathrm{~mL}$ straws, placed $2.5 \mathrm{~cm}$ above the surface of the liquid nitrogen $\left(\mathrm{LN}_{2}\right)$ for $5 \mathrm{~min}$ and plunged in $\mathrm{LN}_{2}$. Thawing was performed in a water bath at $37^{\circ} \mathrm{C}$ for $30 \mathrm{~s}$.

\section{Sperm processing after thawing}

After thawing, each semen sample was divided into three aliquots and submitted to different centrifugation protocols.

\section{Uncentrifuged diluted control (UDC)}

After thawing, sperm was extended in a physiologically balanced solution that would support sperm viability (INRA96; IMV Technologies, L'Aigle, France) to a final concentration of $25 \times 10^{6}$ sperm/mL. Sperm parameters were analyzed as described below.

\section{Sperm washing (SW)}

Sperm was thawed, diluted at the 1:1 ratio and centrifuged $(400 \times \mathrm{g}$ for $7 \mathrm{~min})$. The sperm pellet was resuspended in INRA96 to $25 \times 10^{6}$ sperm $/ \mathrm{mL}$ for sperm analysis.

\section{Single layer centrifugation (SLC)}

Sperm selection was carried out using the colloid Androcoll-E-Small (Swedish University of Agricultural Sciences, Uppsala, Sweden) as described by Ortiz et al. [10]. In short, $2 \mathrm{~mL}$ of thawed sperm were carefully placed on $4 \mathrm{~mL}$ of colloid. The suspension was centrifuged $(300 \times \mathrm{g}$ for $20 \mathrm{~min})$ and the pellet was resuspended in INRA96 to a final concentration of $25 \times 10^{6}$ sperm $/ \mathrm{mL}$. Then, sperm parameters were assessed as described in the following.

\section{Sperm analysis after thawing}

\section{Sperm motility and membrane integrity}

Total (TM, \%) and progressive (PM, \%) sperm motility were evaluated by computer-assisted sperm analysis (CASA) using the Sperm Class Analyzer (SCA 2011 v.5.0.1; Microptic S.L., Barcelona, Spain) with the settings described by Ortiz et al. [13]. Membrane integrity was assessed using Vital Test kit (Halotech DNA, Madrid, Spain) following the manufacturer's instructions [10]. Red-stained sperm were considered as membrane-damaged and green sperm were considered as membrane-intact sperm (MIS, \%).

\section{Sperm DNA fragmentation (sDF) analysis}

The degree of DNA damage in each sample was quantified using the sperm DNA fragmentation index. This parameter was assessed using the Halomax kit (Halotech DNA SL, Madrid, Spain) as previously described by Ortiz et al. [10]. This test is based on the dispersion of the chromatin (halo) after an exposure to a lysing solution. In order to evidence the halos of chromatin, samples were stained with a commercial kit for green fluorescence (Halotech DNA SL). Those sperm with large halos (at least double diameter than the core) were considered to have fragmented DNA. At least 300 spermatozoa per sample were counted and the percentage of fragmented DNA cells was recorded (sDF, \%).

\section{Experimental design}

\section{Experiment 1: Effect of UDC, SW and SLC on DNA fragmentation dynamics}

A dynamic assessment of DNA fragmentation was carried out by incubating an aliquot from UDC, SW and SLC samples for $24 \mathrm{~h}$ at $37^{\circ} \mathrm{C}$. The sDF was evaluated 
at T0 (baseline), T3, T6 and T24 h and compared between and within treatments.

\section{Experiment 2: Comparison between the coefficient of determinations $\left(R^{2}\right)$ of linear, exponential and polynomial regression in SDF dynamics}

The accuracy of three different regression models (linear, exponential, and polynomial) was evaluated by comparing the coefficient of determination $\left(R^{2}\right)$. Then, sDF dynamics were compared among treatments using the most accurate regression model.

\section{Statistical analysis}

In Experiment 1 statistical analysis was performed using the Statistical Analysis Systems software (SAS v.9.0; SAS Institute Inc., Cary, NC, USA). A general linear model (PROC MIXED) with animals, ejaculates, treatments and time as fixed effects was performed. Differences between treatments (UDC vs. SW vs. SLC) and times (T0 vs. T3 vs. T6 vs. T24) were assessed.

In Experiment 2, sDF (\%) values ( $y$ coefficient) at 0, 3, 6 and $24 \mathrm{~h}$ ( $x$ coefficient) were adjusted to linear, exponential and second order polynomial models. The $\mathrm{R}^{2}$ was calculated for each replicate and model using Microsoft Excel for Mac v.14 (Microsoft Corporation, Redmond, WA, USA) and $\mathrm{R}^{2}$ was compared separately for each treatment (UDC, SW and SLC), among models (linear, exponential and polynomial) with PROC MIXED (SAS) using animals and ejaculates as fixed effects.

Since second order polynomial functions are parabolic lines, the derivative function $\frac{d s D F}{d t}\left(\frac{\%}{h}\right)$ was calculated for each treatment (UDC, SW and SLC). Afterwards, a graphic was represented using the rate of change of sDF $(\% / h$, DNA fragmentation velocity) of the polynomial function $\frac{d s D F}{d t}\left(\frac{\%}{h}\right)$ as $y$ coefficient and time $(0,3,6$ and $24 \mathrm{~h})$ as $x$ coefficient. The slopes of these straight lines (DNA fragmentation acceleration, $\% / \mathrm{h}^{2}$ ) were compared between treatments (UDC vs. SW vs. SLC) by ANCOVA using GraphPad Prism v.6 for Mac OS v.6 (GraphPad Software, San Diego, CA, USA).

All values were expressed as the mean \pm standard error of the mean (SEM). Significant differences were considered when $P<0.05$. Duncan post hoc test was carried out to assess differences between treatments.

\section{Results}

\section{Sperm parameters after thawing}

Mean values of sperm parameters obtained immediately after thawing were as follows: $\mathrm{TM}=58.31 \pm 4.57, \mathrm{PM}=$ $47.66 \pm 4.07$, MIS $=57.53 \pm 2.71$, and $\mathrm{sDF}=12.98 \pm 1.52$.

\section{Sperm DNA fragmentation (sDF) dynamics}

A comparison between UDC, SW, and SLC after thawing up to $24 \mathrm{~h}$ of incubation at $37^{\circ} \mathrm{C}$ is shown in Fig. 1 . Significantly lower values of sDF $(P<0.001)$ were found for SLC after $24 \mathrm{~h}$ of incubation.

Table 1 illustrates the sDF dynamics over time for each treatment (UDC, SW and SLC). Significantly higher values $(P<0.001)$ of sDF were obtained after $6 \mathrm{~h}$ of incubation for UDC and SW. However, in SLC-selected aliquots there were not significant differences for $24 \mathrm{~h}$ of incubation at $37{ }^{\circ} \mathrm{C}$.

\section{Regression models fit to the data}

The $\mathrm{R}^{2}$ for SDF dynamics was significantly higher $(P=$ 0.001) in polynomial regression models in comparison to linear and exponential models for UDC $(0.9699 \pm$ 0.0087 vs. $0.8694 \pm 0.0335$ vs. $0.8014 \pm 0.343$ ), $\quad \mathrm{SW}$ $(0.9667 \pm 0.0120$ vs. $0.9324 \pm 0.0190$ vs. $0.8828 \pm 0.0251)$, and SLC $(0.9706 \pm 0.0097$ vs. $0.8326 \pm 0.0605$ vs. 0.0826 \pm 0.0581 ) (Fig. 2).

\section{SDF dynamics in polynomial regression}

Figure 3 shows the graphical representation of the polynomial regression models for UDC, SW and SLC.

Since second order polynomial functions are graphically represented with parabolic lines, they cannot be compared with each other as a whole. The derivative of the polynomial function $\frac{d s D F}{d t}\left(\frac{\%}{h}\right)$ is the rate of change of the function (fragmentation rate, \%/h). Figure 4 represents the velocity of fragmentation with respect to time for UDC, SW and SLC. The slopes of these lines are the acceleration of fragmentation (aSDF, fragmentation rate/ time, $\left.\% / h^{2}\right)$. Significant differences between the slopes of UDC $(-0.0683 \pm 0.0265)$, SW $(0.0106 \pm 0.0130)$ and SLC $(-0.0073 \pm 0.0141)$ were obtained $(P=0.0141)$.

\section{Discussion}

The results of this study indicate that DNA in frozenthawed donkey sperm selected by SLC is more resistant to a stressor (incubation at $37^{\circ} \mathrm{C}$ up to $24 \mathrm{~h}$ ) than control or SW. In order to compare SDF values obtained after each centrifugation procedure (UDC, SW and SLC), semen samples were submitted to incubation at $37{ }^{\circ} \mathrm{C}$ for $24 \mathrm{~h}$. Afterwards, a static and a dynamic assessment of the DNA fragmentation dynamics were carried out. The static analysis of the sDF dynamics, which consisted of a comparison of treatments (UDC vs. SW vs. SLC) immediately finishing the centrifugation protocols (T0) and after $3 \mathrm{~h}$ (T3), $6 \mathrm{~h}$ (T6) and $24 \mathrm{~h}$ (T24) of incubation at $37{ }^{\circ} \mathrm{C}$. No differences in sDF values were seen between treatments up to $24 \mathrm{~h}$. The stability of the DNA molecule in each centrifugation protocol (T0 vs. T3 vs. T6 vs. T24) was evaluated. In this 


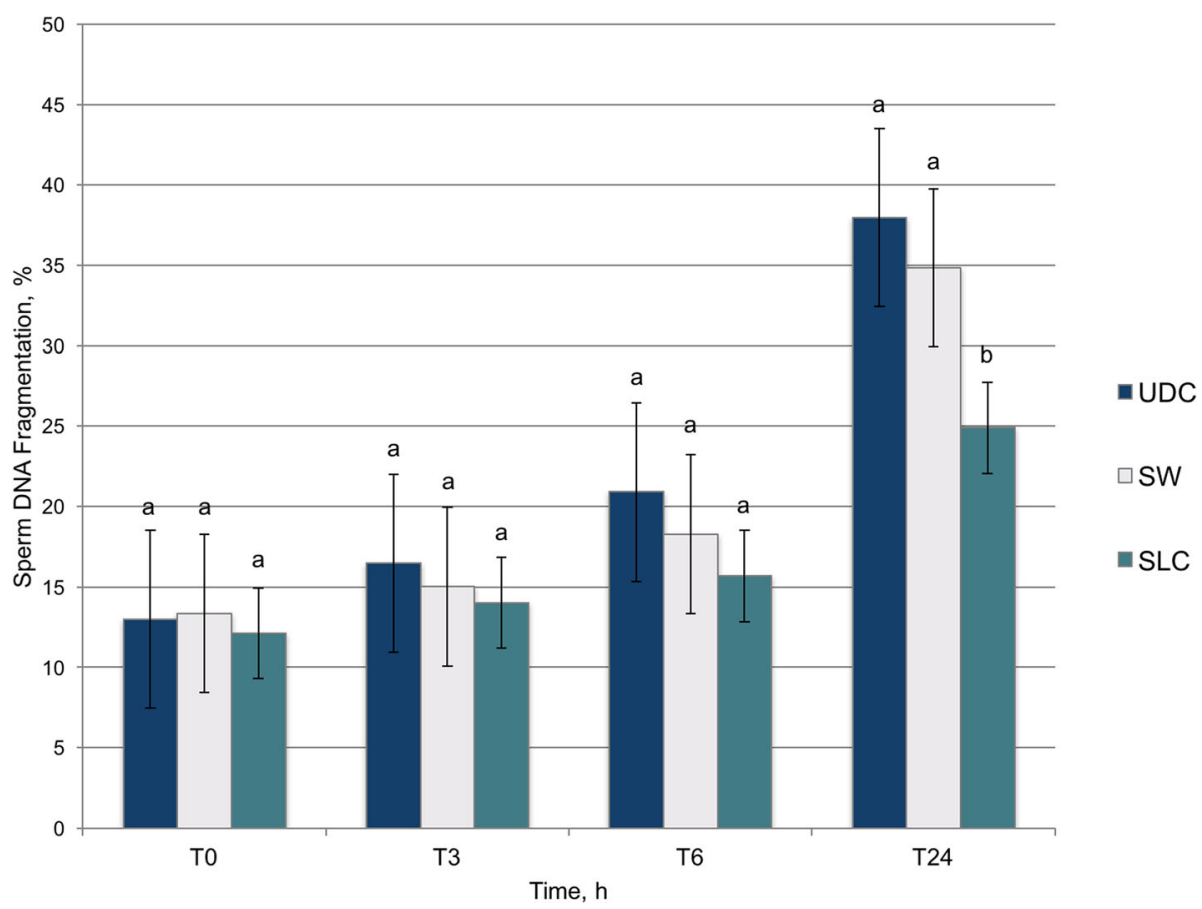

Fig. 1 Effect of centrifugation (UDC, SW, and SLC) on the percentage of sperm DNA fragmentation (SDF, \%) of frozen-thawed donkey sperm for $24 \mathrm{~h}$ of incubation at $37^{\circ} \mathrm{C}$. Values are expressed as means (bars) \pm SEM (error bars). Different superscripts letters indicate significant differences $(P<0.05)$. UDC = Uncentrifuged diluted control; SW = Sperm washing; SLC = Single Layer Centrifugation; TO, T3, T6, T24 = Incubation for $0,3,6,24 \mathrm{~h}$ at $37^{\circ} \mathrm{C}$

regard, sDF values did not increase until $6 \mathrm{~h}$ of incubation for UDC and SW. However, sDF remained stable for up to $24 \mathrm{~h}$ of incubation in SLC samples. Previous studies performed in stallion semen and donkey semen have not found differences in DNA fragmentation baseline values (T0) after colloid centrifugation [10, 14]. However, according to other studies, sDF must be studied dynamically by submitting the semen sample to a stressor in order to find possible cryptic damage in the DNA $[11,12,15]$. When a dynamic assessment was performed, other studies showed that SLC was able to select those stallion sperm more resistant to DNA fragmentation $[16,17]$. Thus, the static and dynamic assessments of sDF dynamics seem to agree that the DNA fragmentation process in SLC samples is slower in comparison to UDC and SW.

Table 1 Effect of time of incubation of sperm at $37^{\circ} \mathrm{C}$ on sperm DNA fragmentation (sDF, \%) within each centrifugation procedure (UDC, SW and SLC)

\begin{tabular}{llllll}
\hline & T0 & T3 & T6 & T24 & $P$-value \\
\hline UDC & $12.98 \pm 1.52^{\mathrm{c}}$ & $16.47 \pm 1.40^{\mathrm{c}}$ & $20.90 \pm 1.34^{\mathrm{b}}$ & $35.86 \pm 3.21^{\mathrm{a}}$ & $<0.001$ \\
SW & $13.35 \pm 1.17^{\mathrm{c}}$ & $15.01 \pm 1.27^{\mathrm{bc}}$ & $18.28 \pm 2.21^{\mathrm{b}}$ & $34.84 \pm 2.60^{\mathrm{a}}$ & $<0.001$ \\
SLC & $11.48 \pm 1.67^{\mathrm{b}}$ & $14.02 \pm 2.16^{\mathrm{b}}$ & $15.70 \pm 2.26^{\mathrm{b}}$ & $24.91 \pm 2.19^{\mathrm{a}}$ & $<0.001$
\end{tabular}

$T 0, T 3, T 6, T 24$ incubation for $0,3,6,24 \mathrm{~h}$ at $37^{\circ} \mathrm{C}, U D C$ uncentrifuged diluted control, SW sperm washing, SLC single layer centrifugation

Values are expressed as mean \pm standard error of the mean (SEM)

Different letters indicate significant differences
Dynamic processes (such as sperm DNA fragmentation) are commonly used in biology as they provide insight into how a force (e. g. incubation at $37^{\circ} \mathrm{C}$ over time) acts to change a cell, an organism, a population, or an assemblage of species [18]. Since we want to know how sDF changes over time $(t)$, that is, $s D F(t)$, a dynamic model is appropriate. There are two main types of dynamic models, "discrete time" and "continuous time", depending on whether time is represented in discrete steps or along continuous axis. In practice, it is not possible to evaluate sDF continually: instead, we must divide the sample into aliquots at intervals of time. Thus, it is crucial to choose a suitable time scale for our study. Previous reports in donkeys [19] and horses [12] have shown that significant changes in sDF between treatments and individuals occurred from 6 to $24 \mathrm{~h}$ of incubation at $37^{\circ} \mathrm{C}$ when using a chromatin dispersion test, or $4 \mathrm{~h}$ of incubation for the sperm chromatin structure assay (SCSA) by flow cytometry [20]. However, since one of the objectives of this study was to fine-tune a model, in order to adjust the model to reality, we needed to have as many points as possible. Therefore, we set the points at 0 , 3, 6 and $24 \mathrm{~h}$ of incubation.

Once the variable (sDF) and the units of time $(0,3,6$ and $24 \mathrm{~h}$ ) are stablished, their relationship was evaluated. The most common single-variable functions or mathematical models which regularly arise in all areas of biology are linear, exponential and polynomial. It is common to think of a relationship between two variables as 

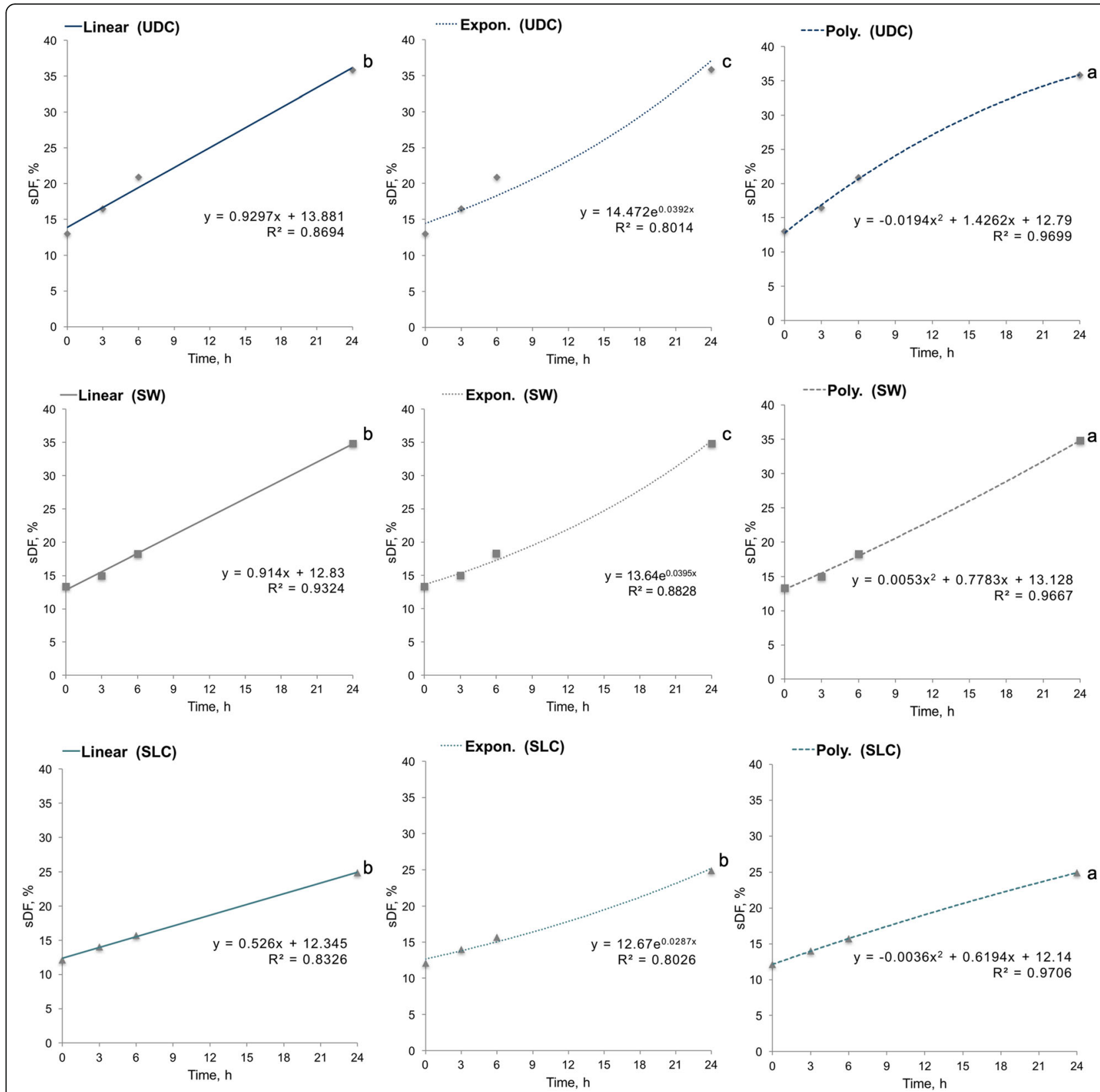

Fig. 2 Coefficient of determination $\left(R^{2}\right)$ for linear, exponential and polynomial regression models within treatments (UDC, SW, SLC). Different letters indicate significant differences $(P<0.05)$. sDF = Sperm DNA fragmentation; UDC = Uncentrifuged diluted control; SW = Sperm washing; SLC = Single layer centrifugation; Expon. $=$ Exponential; Poly. $=$ Polynomial

a straight line; in fact, it has been taken for granted that $\mathrm{sDF}$ follows a linear regression equation

$$
y=f(x)=a x+b
$$

where $y=\operatorname{sDF}(\%), x=$ time $(\mathrm{h}) ; a=$ fragmentation rate $(\% / \mathrm{h})$; $b=$ intercept (\%). This model has been applied to calculate the $\mathrm{sDF}$ rate $(\mathrm{rSDF})$ using the slope of the linear regression line. Nonetheless, a linear regression model, as its name indicates, is a straight line. This would mean that rSDF is constant over time which causes some limitations. In this sense, a phenomenon called the "Plateau effect" has been previously described when using linear regression to assess sDF dynamics in stallion sperm [12]. This so-called Plateau effect is a change in the slope of the regression line, due to a change in the velocity of fragmentation. This singularity leads to confounding results if the equation is not divided into two different lines, representing $0-6 \mathrm{~h}$ and $6-24 \mathrm{~h}$ of incubation. Nonetheless, that provisional adjustment shows that the sDF dynamics does not fit a linear regression equation. 


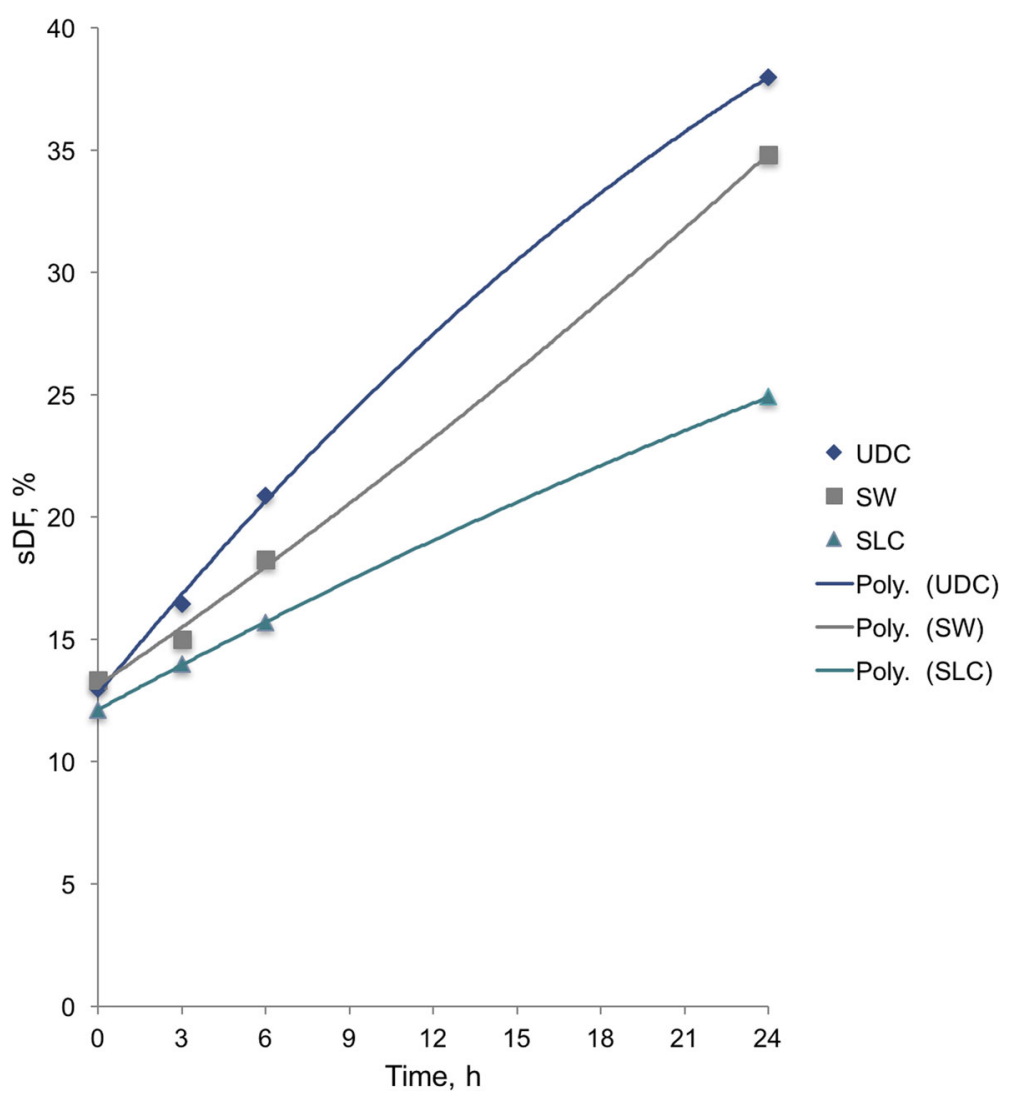

Fig. 3 Polynomial regression lines for UDC, SW and SLC. sDF = Sperm DNA fragmentation; UDC = Uncentrifuged diluted control; SW = Sperm washing; SLC = Single layer centrifugation; Poly $=$ = Polynomial

Non-linear equations are those which can be represented as curves. In this sense, exponential and polynomial functions are very popular for modelling biological functions. Exponential function is probably one of the most important function in dynamic models of biology, it is represented as

$$
y=f(x)=b \cdot e^{a x}
$$

its main application is describing fast growth [21]. The exponential model has previously been applied to explain the behavior of sDF dynamics in human ejaculates [22]; nevertheless, this explanation was merely descriptive since there was no statistical comparison between other mathematical models. Last but not least, second order polynomial function (or quadratic),

$$
y=f(x)=a x^{2}+b x+c ;
$$

has a wide variety of important uses in biology. It describes the rate of growth when resources are limited [23]. Although previous studies have shown sDF curves which might fit a polynomial model [24, 25], to date, no study has tried to explained sDF dynamics using this model. Actually, to the best of the authors' knowledge, a fine-tuning of models has never been performed for sDF dynamics. Furthermore, this quadratic model explains by itself the behavior of sDF from 0 to $24 \mathrm{~h}$ of incubation, without the need to split the $0-6 \mathrm{~h}$ and $6-24 \mathrm{~h}$ as it occurs with the traditional assessment by linear regression [12].

On the whole, mathematical models are mainly used to make predictions about the behavior of a variable at any time. Logically, the closer to reality the model is the more accurate the prediction will be. In statistics, $R^{2}$ provides a measure of how well observed outcomes are replicated by the model, based on the proportion of total variation of outcomes explained by the model [26]. Surprisingly, second order polynomial achieved the significantly highest $R^{2}$ in all the treatments studied (UDC, SW and SLC), becoming a more accurate model than linear regression or exponential to predict SDF over time. The more than acceptable $\mathrm{R}^{2}$ mean values obtained for UDC, SW and SLC (0.9699, 0.9667 and 0.9706 , respectively) indicate that the conclusions obtained from this model are very close to reality.

The fact that our model is a parabola implies that sDF rate is not constant, which also means that there is $\mathrm{sDF}$ acceleration, an observation that has never been 


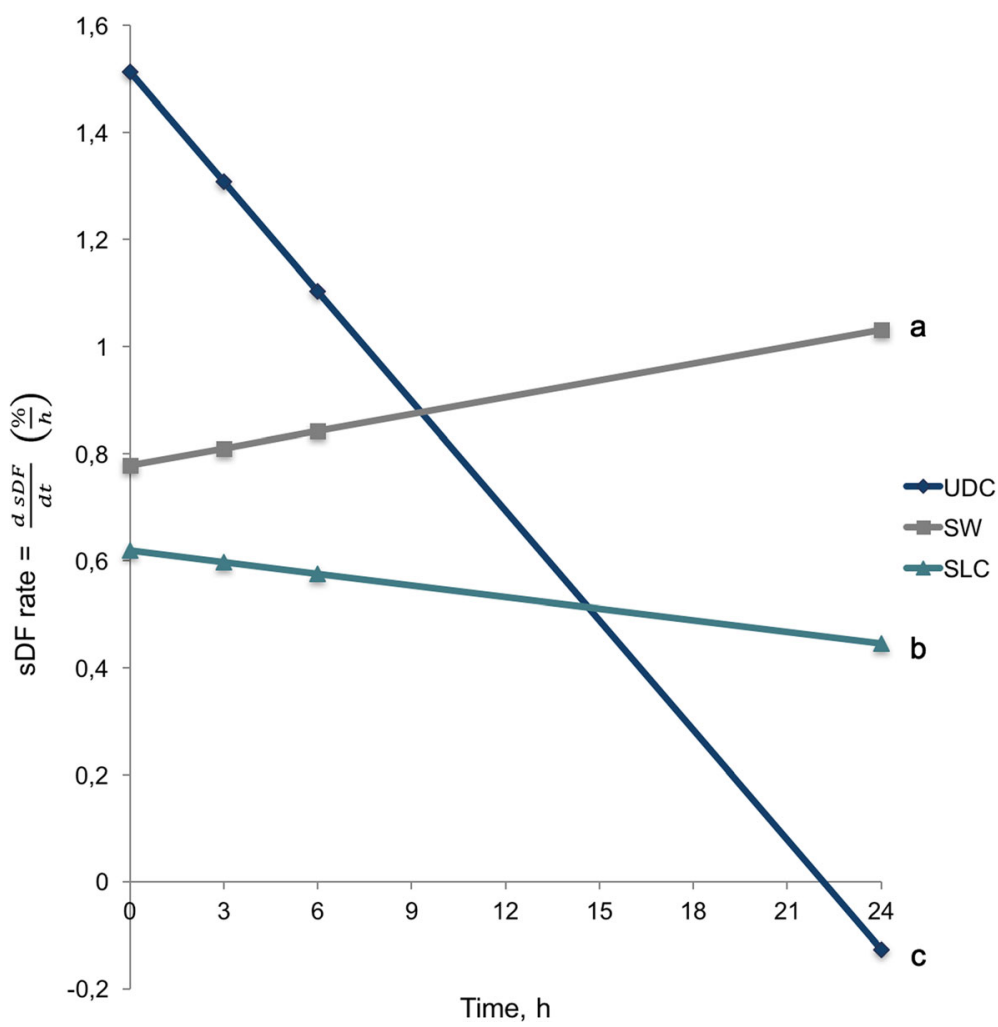

Fig. 4 Sperm DNA fragmentation rate $(\% / h)$ in relation to time of incubation at $37{ }^{\circ} \mathrm{C}$ for UDC, SW and SLC. Different letters indicate significant differences between slopes $(P<0.05)$. sDF = Sperm DNA fragmentation; UDC = Uncentrifuged diluted control; SW = Sperm washing; SLC = Single layer centrifugation

described before. In quadratic functions, we can track the rate of change of the function using a difference equation. A difference equation specifies how much a variable changes from one time unit to the next. In quadratic functions, the rate of change over time is expressed as follows:

$$
y^{\prime}=f^{\prime}(x)=\frac{d y}{d x}=2 a x+b
$$

in our function, the rate of change of the function is the rate of change of rSDF. Therefore, sDF rate (rSDF) in quadratic functions is expressed as

$$
r S D F=\frac{d s D F}{d t}\left(\frac{\%}{h}\right)
$$

and the fragmentation acceleration (aSDF) is the rate of change over time, or the slope of the derivative of the quadratic function:

$$
a S D F=\frac{d s D F / d t}{t}\left(\frac{\%}{h^{2}}\right) .
$$

Surprisingly, when representing the rates of change for UDC, SW and SLC, the three lines obtained were very different. Although UDC showed faster rSDF for about $10 \mathrm{~h}$, it was also the treatment with significantly lower acceleration $\left(a S D F_{U D C}=-0.0683 \pm 0.0265\right)$; this marked deceleration explains the Plateau effect described in stallions. In SW samples, rSDF values were lower than UDC until $10 \mathrm{~h}$ of incubation, but a higher acceleration $\left(a S D F_{S W}=0.0106 \pm 0.0130\right)$ increased rSDF from that point on. The SLC also showed a negative acceleration $\left(a S D F_{S L C}=-0.0073 \pm 0.0141\right)$, but not as marked as in UDC samples. On the one hand, centrifuged samples after thawing (SW and especially SLC) showed lower values than control (UDC) for 10 and $15 \mathrm{~h}$, respectively. On the other hand, centrifugation increased the acceleration of fragmentation, in particular in SW samples. It could be possible that post-thawing centrifugation, mainly SW, damaged the fixing mechanism of the DNA molecule [27, 28]. However, sDF values are lower during the time studied for SW and SLC. In this sense, if DNA fragmentation processes also occur in vivo in a nonlinear manner, the initially rapid fragmentation rate may reduce the effective and fertile sperm concentration before they have a chance to colonize the oviducts. Therefore, delaying the early fragmentation events could be beneficial in this respect and may help to improve fertility. Further studies involving fertility and the relationship between DNA fragmentation timing and the timing of sperm transport and fertilization are needed to characterize the mechanism of action of this damage. 
Nonetheless, we need to keep in mind that rSDF values have been obtained from a model, i.e. they are expected rather than observed values. In order to fit the model even more, further studies with more frequent assessments (between 6 and $24 \mathrm{~h}$ and after $24 \mathrm{~h}$ ) would be needed to obtain more accurate predictions. It is of the utmost importance to fit data to an accurate model in order to know exactly how this molecule behaves. In this sense, DNA cannot have been correlated to any other sperm parameter $[19,29]$ using linear regression to work with SDF dynamics. Hopefully, this study provides new tips so that correlation between DNA fragmentation dynamics and sperm quality is focused from a new perspective.

\section{Conclusions}

SLC after thawing seems to preserve DNA longevity for longer in comparison to UDC and SW. Moreover, the fine-tuning of models has shown that SDF dynamics in frozen-thawed donkey semen fits a second order polynomial model, which implies that fragmentation rate is not constant and fragmentation acceleration must be taken into account to elucidate hidden damage in the DNA molecule.

\begin{abstract}
Abbreviations
aSDF: Sperm DNA fragmentation acceleration; CASA: Computer-assisted sperm analysis; Expon.: Exponential; h: Hours of incubation at $37^{\circ} \mathrm{C}$; $\mathrm{LN}_{2}$ : Liquid nitrogen; min: Minutes; MIS: Membrane intact sperm; PM: Progressive sperm motility; Poly: Second order polynomial; $R^{2}$ : Coefficient of determination; rSDF: Sperm DNA fragmentation rate; s: Seconds; SAS: Statistical Analysis Systems Software; sDF: Sperm DNA fragmentation; SEM: Standard error of the mean; SLC: Single layer centrifugation; SW: Sperm washing; T0-T3-T6-T24: 0-3-6-24 h of incubation at $37^{\circ} \mathrm{C}$; TM: Total sperm motility; UDC: Uncentrifuged diluted control
\end{abstract}

\section{Acknowledgements}

The authors are extremely grateful to Fundación Casa del Burro (Rute, Córdoba, Spain) for providing the animals.

\section{Funding}

This study was partially supported by grants RZ2009-00006-00-00 (Instituto Nacional de Investigación y Tecnología Agraria y Alimentaria, Ministerio de Ciencia e Innovación, Spain) and AGL-2013-42726-R (Secretaría de Estado de Investigación, Desarrollo e Innovación, Ministerio de Economía y Competitividad, Spain). I. Ortiz was supported by a Ph.D. fellowship from the ceiA3 (Andalucía, Spain) with funding provided by Banco Santander through its Global Division, Santander Universidades. J M Morrell is funded by the Swedish Foundation for Equine Research, Stockholm, Sweden (H14-47-008).

\section{Authors' contributions}

IO contributed to all sections. JD and MH contributed to the study design, data analysis and interpretation, preparation of the manuscript and final approval of the manuscript. JMM, JG and FC collaborated in the experimental design. JMM provided the colloid and revised the English. JMJ revised modelling and calculation. All authors read and approved the final manuscript.

\section{Competing interests}

The authors declare that they have no competing interests. J M Morrell is the inventor and one of the patent holders of Androcoll-E and SLC.

\section{Consent for publication}

Not applicable.

\section{Ethics approval}

All animal procedures were performed in accordance with the Spanish laws for animal welfare and experimentation, under the supervision of the Bioethical and Biosafety Committee of the University of Cordoba (UCO).

\section{Author details \\ 'Veterinary Reproduction Group, Department of Animal Medicine and Surgery, Faculty of Veterinary Medicine, University of Cordoba, 14071 Cordoba, Spain. ${ }^{2}$ Department of Clinical Sciences, Division of Reproduction, Swedish University of Agricultural Sciences, Box 7054 SE-75007 Uppsala, Sweden. ${ }^{3}$ Department of Biology, Universidad Autónoma de Madrid, 28049 Madrid, Spain. ${ }^{4}$ Department of Reproduction, Centro Militar de Cría Caballar (FESCCR-Ministry of Defense), 05005 Ávila, Spain. ${ }^{5}$ Department of Chemistry and Physics of Materials, University of Salzburg, Hellbrunner Straße 34/III, A-5020 Salzburg, Austria.}

Received: 1 September 2016 Accepted: 21 February 2017

Published online: 07 March 2017

\section{References}

1. Evenson DP. The Sperm Chromatin Structure Assay $\left(\mathrm{SCS}^{\oplus}\right)$ and other sperm DNA fragmentation tests for evaluation of sperm nuclear DNA integrity as related to fertility. Anim Reprod Sci. 2016;169:56-75.

2. Esteves SC. Novel concepts in male factor infertility: clinical and laboratory perspectives. J Assist Reprod Genet. 2016;33:1319-35.

3. Ozkosem B, Feinstein SI, Fisher AB, O'Flaherty C. Advancing age increases sperm chromatin damage and impairs fertility in peroxiredoxin 6 null mice. Redox Biol. 2015;5:15-23.

4. Ni K, Spiess AN, Schuppe HC, Steger K. The impact of sperm protamine deficiency and sperm DNA damage on human male fertility: a systematic review and meta-analysis. Andrology. 2016;4:789-99.

5. Johnston SD, López-Fernández C, Arroyo F, Gosálbez A, Cortés Gutiérrez El, Fernández J-L, et al. Reduced sperm DNA longevity is associated with an increased incidence of still born; evidence from a multi-ovulating sequential artificial insemination animal model. Reprod Genet. 2016: 33(9):1231-8.

6. Oleszczuk K, Augustinsson L, Bayat N, Giwercman A, Bungum M. Prevalence of high DNA fragmentation index in male partners of unexplained infertile couples. Andrology. 2013;1:357-60.

7. Brinsko SP, Rigby SL, Lindsey AC, Blanchard TL, Love CC, Varner DD. Pregnancy rates in mares following hysteroscopic or transrectally-guided insemination with low sperm numbers at the utero-tubal papilla. Theriogenology. 2003:59:1001-9.

8. Morrell JM, Johannisson A, Dalin AM, Hammar L, Sandebert T, RodriguezMartinez H. Sperm morphology and chromatin integrity in Swedish warmblood stallions and their relationship to pregnancy rates. Acta Vet Scand. 2008;50:2.

9. Ortiz I, Dorado J, Acha D, Gálvez MJ, Urbano M, Hidalgo M. Colloid single-layer centrifugation improves post-thaw donkey (Equus asinus) sperm quality and is related to ejaculate freezability. Reprod Fertil Dev. 2015;27:332-40

10. Ortiz I, Dorado J, Morrell JM, Crespo F, Gosálvez J, Gálvez MJ, et al. Effect of single-layer centrifugation or washing on frozen-thawed donkey semen quality: Do they have the same effect regardless of the quality of the sample? Theriogenology, 2015:84:294-300

11. Gosalvez J, Lopez-Fernandez C, Fernandez JL, Gouraud A, Holt WV. Relationships between the dynamics of iatrogenic DNA damage and genomic design in mammalian spermatozoa from eleven species. Mol Reprod Dev. 2011;78:951-61.

12. Lopez-Fernandez C, Crespo F, Arroyo F, Fernandez JL, Arana P, Johnston SD, et al. Dynamics of sperm DNA fragmentation in domestic animals 11. The stallion. Theriogenology. 2007;68:1240-50

13. Ortiz I, Dorado J, Ramírez L, Morrell JM, Acha D, Urbano M, et al. Effect of single layer centrifugation using Androcoll-E-Large on the sperm quality parameters of cooled-stored donkey semen doses. Animal. 2014:8:308-15.

14. Costa AL, Martins-Bessa A, de Andrade AR, Guimaraes T, Rebordao MR, Gaivao I, et al. Single Layer Centrifugation with Androcoll-ETM improved progressive motility and percentage of live spermatozoa with intact acrosome of chilled stallion semen but did not have an effect on DNA integrity. Open J Anim Sci. 2012;2:159-65. 
15. Gosalvez J, Fernandez UL, Gosalbez A, Arrollo F, Agarwal A, Lopez-Fernandez C. Dynamics of sperm DNA fragmentation in mammalian species as assessed by the SCD methodology. Fertil Steril. 2007;88:S365.

16. Crespo F, Gosalvez J, Gutiérrez-Cepeda L, Serres C, Johnston SD. Colloidal centrifugation of stallion semen results in a reduced rate of sperm DNA fragmentation. Reprod Domest Anim. 2013;48:e23-5.

17. Gutiérrez-Cepeda L, Fernández Á, Crespo F, Ramírez MÁ, Gosálvez J, Serres C. The effect of two pre-cryopreservation single layer colloidal centrifugation protocols in combination with different freezing extenders on the fragmentation dynamics of thawed equine sperm DNA. Acta Vet Scand. 2012;54:72.

18. Otto SP, Day T. A biologist's guide to mathematical modeling in ecology and evolution. Princenton: Princenton University Press; 2007.

19. Cortes-Gutierrez El, Crespo F, Gosalvez A, Davila-Rodriguez MI, LopezFernandez C, Gosalvez J. DNA fragmentation in frozen sperm of Equus asinus: Zamorano-Leones, a breed at risk of extinction. Theriogenology. 2008;69:1022-32.

20. Sabatini C, Mari G, Mislei B, Love C, Panzani D, Camillo F, et al. Effect of post-thaw addition of seminal plasma on motility, viability and chromatin integrity of cryopreserved donkey jack (Equus asinus) spermatozoa. Reprod Domest Anim. 2014;49:989-94.

21. Ferretti AC, Joyce GF. Kinetic properties of an RNA enzyme that undergoes self-sustained exponential amplification. Biochemistry. 2013;52:1227-35.

22. Gosalvez J, Nunez R, Fernandez JL, Lopez-Fernandez C, Caballero P. Dynamics of sperm DNA damage in fresh versus frozen-thawed and gradient processed ejaculates in human donors. Andrologia. 2011:43:373-7.

23. Martín-Landrove R, Guillén N, Martín-Landrove M. A kinetic model for tumor survival curves: its relation to the linear-quadratic model. 3rd ed. Munich: World Congress on Medical Physics and Biomedical Engineering: Radiation Protection and Dosimetry, Biological Effects of Radiation; 2009. p. 508-11.

24. Gosálvez J, López-Fernández C, Hermoso A, Fernández JL, Kjelland ME. Sperm DNA fragmentation in zebrafish (Danio rerio) and its impact on fertility and embryo viability — Implications for fisheries and aquaculture. Aquaculture. 2014;433:173-82.

25. Gosalvez J, Ramirez MA, Lopez-Fernandez C, Crespo F, Evans KM, Kjelland $M E$, et al. Sex-sorted bovine spermatozoa and DNA damage: II. Dynamic features. Theriogenology. 2011;75:206-11.

26. Colin Cameron A, Windmeijer FAG. An R-squared measure of goodness of fit for some common nonlinear regression models. J Econ. 1997;77:329-42.

27. Gunes S, Al-Sadaan M, Agarwal A. Spermatogenesis, DNA damage and DNA repair mechanisms in male infertility. Reprod BioMed Online. 2015;31:309-19.

28. Marcon L, Boissonneault G. Transient DNA strand breaks during mouse and human spermiogenesis:New insights in stage specificity and link to chromatin remodeling. Biol Reprod. 2004;70:910-8.

29. Lewis SEM. Should sperm DNA fragmentation testing be included in the male infertility work-up? Reprod BioMed Online. 2015;31:134-7.

\section{Submit your next manuscript to BioMed Central and we will help you at every step:}

- We accept pre-submission inquiries

- Our selector tool helps you to find the most relevant journal

- We provide round the clock customer support

- Convenient online submission

- Thorough peer review

- Inclusion in PubMed and all major indexing services

- Maximum visibility for your research

Submit your manuscript at www.biomedcentral.com/submit
Biomed Central 